\title{
Generic Pest Risk Analysis for Potato in Nepal
}

\author{
Baidya Nath Mahto ${ }^{\circledR}$ \\ Plant Pathology Division, NARC, Khumaltar, Lalitpur, @: bnmahto 7@yahoo.com
}

Received June 2015, Revised August 2016, Accepted January 2017, Published May 2017

Scientific Editors: Samudra Lal Joshi, Ram Devi Timila, Tek B. Gurung

Copyright @2017 NARC. Permits unrestricted use, distribution and reproduction in any medium provided the original work is properly cited

\begin{abstract}
Pest Risk Analysis (PRA) is the process of evaluation for biological and economic evidences in order to determine whether a pest should be regulated under phyto-sanitary measures. The present mini review highlights the potential potato pathogen list recorded in Nepal harmful for potato production and productivity. At global scale altogether 135 potential quarantine pests (PQP) for potato has been recorded, while in Nepal only 92 PQP were recorded. Out of those 52, 13 and 27 were fungi, bacteria and viruses respectively. Among the 92 PQP, 34,30 and 13 were considered at high, medium and lower risk type pathogens for potato. There was no information available on other $15 \mathrm{PQP}$.
\end{abstract}

Keywords: Pest Risk Analysis, Potential Quarantine Pest, Pathogen, Pest risk assessment

सारांश

शत्रुजीव जोखिम विश्लेषण (PRA) एउटा बैज्ञानिक प्रक्रिया हो । जसमा शत्रुजीवको जैविक तथा आर्थिक तथ्यको आधारमा बाहिरी देशबाट आप्नो देशमा भित्राउन बिरुवा स्वस्थता सम्बन्धी नियमन गर्नु पर्ने हो होइन निर्क्यौल गरिन्छ। प्रस्तुत सक्षिप्त आलेखमा नेपालमा आलुबालीका सभाव्य जोखिमपूर्ण जीवाणुहरुको विवेचनात्मक सुची प्रस्तुत गरिएको छ। संसारभरि आलु बालीमा लाग्ने १३५ थरिरोगका जीवाणुहरु (Pathogens/Pests) सूचीकृत छन् । विश्वब्यापीरुपमा १३५ थरिका जीवाणुहरुलाई सम्भ्याव्य जोखिमयुक्त मानिएका छन्। तथापि, यी रोगका जीवाणुहरुको नेपाली सन्दर्भमा आर्थिक विश्लेषण गर्दा ९२ वटा जीवाणुहरुलाई सम्भाव्य शत्रुजीव (Potential Quarantine Pest, PQP) को रुपमा लिन सकिन्छ । ९२ वटा (PQP) मध्ये पू२, १३ र २७ वटा ढु "सी, विशाणु तथा जीवाणु कमश: रहेका छन् । यी ९२ वटा शत्रुजीव (Pathogen) मध्ये ३४ थरिलाई उच्च, ३० मध्यम, १३ लाई सामान्य जोखिमयुक्त शत्रुजीव मानिएका पाइए। बाँकी १४ वटा शत्रुजीवको जोखिम विश्लेषण सम्बन्धी जानकारी उपलब्ध छैन।

\section{INTRODUCTION}

According to the World Trade Organization (WTO)'s sanitary and quarantine (SPS) agreement on the sharing of the material under Pest Risk Analysis (PRA) is important (Jha et al 2006, Ullah 2016). Sanitary and Phytosanitary (SPS) Agreement of the World Trade Organization (WTO) suggested to implement a Pest Risk Analysis (PRA) while sharing genetic materials, because, it is necessary to develop a comprehensive PRA in international trade and commerce brought out for crops export and import (MoAD 2013). Food and Agriculture Organization (FAO) has taken the initiative to strengthen the plant quarantine and institutional capacity of quality quarantine services in Nepal. Pest risk analysis involves the merging of scientific assessment, ongoing consultation and practical pest management. Nepal being a member of World Trade Organization (WTO) needs to declare the prevailing diseases (pest) status of crops, which has been a major international concern for trade promotion. Pest risk assessment is a process of identifying plant pests as potential hazards and characterizing their risk (MoAD 2013). Risk characterization is risk assessment process of estimating the likelihood or probability of experiencing the adverse effects of pests.

Risk assessment provides the scientific basis for analyses. Its emphasis is on biology, but it also includes a description of economic implications (Ullah 2016). Since the PRA is a recent requirement and obligation to reduce the impact of pathogen. Therefore, the purpose of the paper is to elucidate on potential quarantine pests (PQP) such as fungi, bacteria, viruses and risk of entry, spread, damage etc. 


\section{MATERIALS AND METHODS}

To perform generic pest risk analysis (PRA) Crop Protection Compendium (CPC 2006) provides reference for information on global pathogens (pests) list. The relevant literature was reviewed and the lists of pathogens (disease) have been prepared. Several magazines, journals, proceedings, research report and books with a description of the work performed in Nepal have been consulted. The information gathered has been further upgraded.

\section{Guidelines for rating entry}

Rating= low: The probability of entry is low given the combination of factors including the distribution of the pest source, management practices, low commodity volume, low probability of pest survival in transit, or low probability of contact with susceptible hosts given the intended use. The probability of entry is low but clearly possible given the expected combination of factors.

Rating = medium: Pest entry into in an area is likely given the combination of factors described above. Rating =high: Pest entry is very likely or certain given the combination of factors described above.

Table 1. List of global, Nepal and Potential Quarantine Pest (PQP) for Nepal

\begin{tabular}{|c|c|c|c|}
\hline SN & $\begin{array}{l}\text { Global list on potato pathogen } \\
\text { (Fungi, bacteria, phytoplasma \& viruses) }\end{array}$ & $\begin{array}{l}\text { Updated Nepal pest list } \\
\text { (Fungi, bacteria and viruses) }\end{array}$ & $\begin{array}{l}\text { Potential Quarantine Pest (PQP) } \\
\text { (Fungi, bacteria and viruses) }\end{array}$ \\
\hline 1. & Aecidium cantense (Aecidium potato rust) & Alternaria alternata (alternaria leaf spot) & Aecidium cantense (Aecidium potato rust) \\
\hline 2. & Alternaria alternata (alternaria leaf spot) & Alternaria solani & Alternaria radicina (black rot of carrots) \\
\hline 3. & Alternaria radicina (black rot of carrots) & Alternaria tenuissima & Arthrinium phaeospermum \\
\hline 4. & Alternaria solani (early blight of potato and tomato) & Cercospora solanicola & Bacillus polymyха (bacterial fruit rot) \\
\hline 5. & Arthrinium phaeospermum & Cladosporium tenuissimum & Belicobasidium purpureum Pat. \\
\hline 6. & Aspergillus niger (Aspergillus ear rot) & Phytophthora infestans & Botryotinia fuckeliana (Botrytis rot) \\
\hline 7. & Bacillus polymyxa (bacterial fruit rot) & Synchytrium endobioticum & Burkholderia cepacia (slippery skin of onion) \\
\hline 8. & Belicobasidium purpureum Pat. & Thanatephorus cucumeris (Frank) Donk & Burkholderia gladioli pv. Gladioli (corm scab) \\
\hline 9. & Botryotinia fuckeliana (Botrytis rot) & Rhizoctonia solani & Chalara elegans (black root rot) \\
\hline 10. & Burkholderia cepacia (slippery skin of onion) & Aspergillus niger (Aspergillus ear rot) & Choanephora cucurbitarum (Choanephora blossom blight) \\
\hline 11. & Burkholderia gladioli pv. Gladioli (corm scab) & Corticium rolfsii (collar rot) & Cochliobolus australiensis \\
\hline 12. & Cercospora solanicola & Fusarium coeruleum (dry rot: potato) & Cochliobolus hawaiiensis (leaf spot: maize) \\
\hline 13. & Chalara elegans (black root rot) & Cercospora solani-tuberosi & Coleosporium tussilaginis f.sp. senecionis-sylvati \\
\hline 14. & Choanephora cucurbitarum (Choanephora blossom blight) & Cercospora concors & Colletotrichum coccodes (anthracnose of tomato) \\
\hline 15. & Cladosporium tenuissimum & Spongospora subterranean & Didymella bryoniae (gummy stem blight) \\
\hline 16. & Clavibacter michiganensis subsp. Sepedonicus (Potato ring rot) & Cochliobolus lunatus (Cabage, Rice) & Didymella lycopersici (canker of tomato) \\
\hline 17. & Cochliobolus australiensis & Sclerotinia sclerotiorum (collar rot) Lentil & $\begin{array}{l}\text { Erwinia carotovora subsp. Carotovora (bacterial root rot of } \\
\text { sweet potato) }\end{array}$ \\
\hline 18. & Cochliobolus hawaiiensis (leaf spot: maize) & Colletotrichum capsici (leaf spot of peppers) & Erwinia chrysanthemi \\
\hline 19. & Cochliobolus lunatus (black kernel of rice) & Septoria lycopersici (leaf spot of tomato)Tomato & $\begin{array}{l}\text { Erwinia chrysanthemi pv. Chrysanthemi (bacterial maize } \\
\text { stalk rot) }\end{array}$ \\
\hline 20. & Coleosporium tussilaginis f.sp. senecionis-sylvati & Erwinia chrysanthemi pv. Zeae (bacterial stalk rot)Maize & Erysiphe orontii (powdery mildew) \\
\hline 21. & Colletotrichum capsici (leaf spot of peppers) & Pythium aphanidermatum (damping-off)Maize & Fusarium \\
\hline 22. & Colletotrichum coccodes (anthracnose of tomato) & Macrophomina phaseolina (ashy stem blight)Mango & Fusarium culmorum (culm rot: cereals) \\
\hline 23. & Corticium rolfsii (collar rot) & Clavibacter michiganensis subsp. Sepedonicus & Fusarium oxysporum f.sp. tuberose \\
\hline 24. & Didymella bryoniae (gummy stem blight) & Erwinia carotovora subsp. Atroseptica & Geotrichum candidum (sour rot: Citrus spp.) \\
\hline 25. & Didymella lycopersici (canker of tomato) & Ralstonia solanacearum & Gibberella avenacea (bud rot) \\
\hline 26. & Erwinia carotovora & Streptomyces scabiei & Gibberella baccata (biocontrol: velvetleaf) \\
\hline 27. & Erwinia carotovora subsp. Atroseptica (potato blackleg disease) & Potato virus $M$ & Gibberella zeae (cobweb disease) \\
\hline 28. & $\begin{array}{l}\text { Erwinia carotovora subsp. Carotovora (bacterial root rot of } \\
\text { sweet potato) }\end{array}$ & Potato virus $S$ & Helminthosporium solani (silver scurf) \\
\hline 29. & Erwinia chrysanthemi & Potato virus $X$ (potato interveinal mosaic) & Hypocrea rufa (fruit rot: Citrus spp.) \\
\hline 30. & $\begin{array}{l}\text { Erwinia chrysanthemi pv. Chrysanthemi (bacterial maize stalk } \\
\text { rot) }\end{array}$ & Potato virus $Y$ (potato mottle) & Leveillula taurica \\
\hline 31. & Erwinia chrysanthemi pv. Zeae (bacterial stalk rot) & Potato virus A (potato mild mosaic) & Melanotus communis (wireworm, common) \\
\hline 32. & Erysiphe orontii (powdery mildew) & Potato aucuba mosaic virus & Nectria haematococca (dry rot of potato) \\
\hline 33. & Fusarium & Potato leafroll virus & Pectobacterium chrysanthemi (slow wilt) \\
\hline 34. & Fusarium coeruleum (dry rot: potato) & Phytophthora capsici (soft rot of cucurbit fruits) & Phacidiopycnis tuberivora (dryrot: potato) \\
\hline 35. & Fusarium culmorum (culm rot: cereals) & Leveillula taurica (In onion) & Phialophora parasitica (wilt: date palm) \\
\hline 36. & Fusarium oxysporum f.sp. tuberosi & Erwinia chrysanthemi (in onion) & Phoma andigena (black blight of potatoes) \\
\hline 37. & Geotrichum candidum (sour rot: Citrus spp.) & Tomato spotted wilt virus (tomato spotted wilt) in lentil & Phoma eupyrena \\
\hline 38. & Gibberella avenacea (bud rot) & Ralstonia solanacearum race 1 (in tomato) & Phoma exigua var. exigua (leaf blotch) \\
\hline 39. & Gibberella baccata (biocontrol: velvetleaf) & Ralstonia solanacearum race 3 (in tomato) & Phoma foveata (potato gangrene) \\
\hline 43. & Leveillula taurica & Alfalfa mosaic virus (alfalfa yellow spot) & Phytophthora erythroseptica var. erythroseptica (pink rot) \\
\hline 44. & Macrophomina phaseolina (ashy stem blight) & Citrus exocortis viroid (citrus exocortis) & Phytophthora megasperma (apoplexy) \\
\hline 45. & Melanotus communis (wireworm, common) & Tobacco mosaic virus (tobacco mosaic) & Polyscytalum pustulans (skin spot of potato) \\
\hline 46. & Nectria haematococca (dry rot of potato) & & Potato purple-top wilt phytoplasma \\
\hline 47. & Nectria haematococca (dry rot: potato) & & Potato stolbur phytoplasma \\
\hline 48. & Pectobacterium chrysanthemi (slow wilt) & & Potato witches' broom phytoplasma \\
\hline 49. & Phacidiopycnis tuberivora (dryrot: potato) & & Pseudomonas fluorescens (biocontrol: Damping off (cotton)) \\
\hline 50. & Phialophora parasitica (wilt: date palm) & & $\begin{array}{l}\text { Pseudomonas marginalis pv. Marginalis (lettuce marginal } \\
\text { leaf blight) }\end{array}$ \\
\hline 51. & Phoma andigena (black blight of potatoes) & & Pseudomonas putida (biocontrol: Erwinia spp.) \\
\hline 52. & Phoma eupyrena & & Pseudomonas syringae pv. Garcae (bacterial blight of coffee) \\
\hline 53. & Phoma exigua var. exigua (leaf blotch) & & Pseudomonas syringae pv. Tabaci (angular leaf spot) \\
\hline 54. & Phoma foveata (potato gangrene) & & Puccinia pittieriana (common rust of potato) \\
\hline 55. & Phytophthora capsici (soft rot of cucurbit fruits) & & Pythium butleri \\
\hline
\end{tabular}




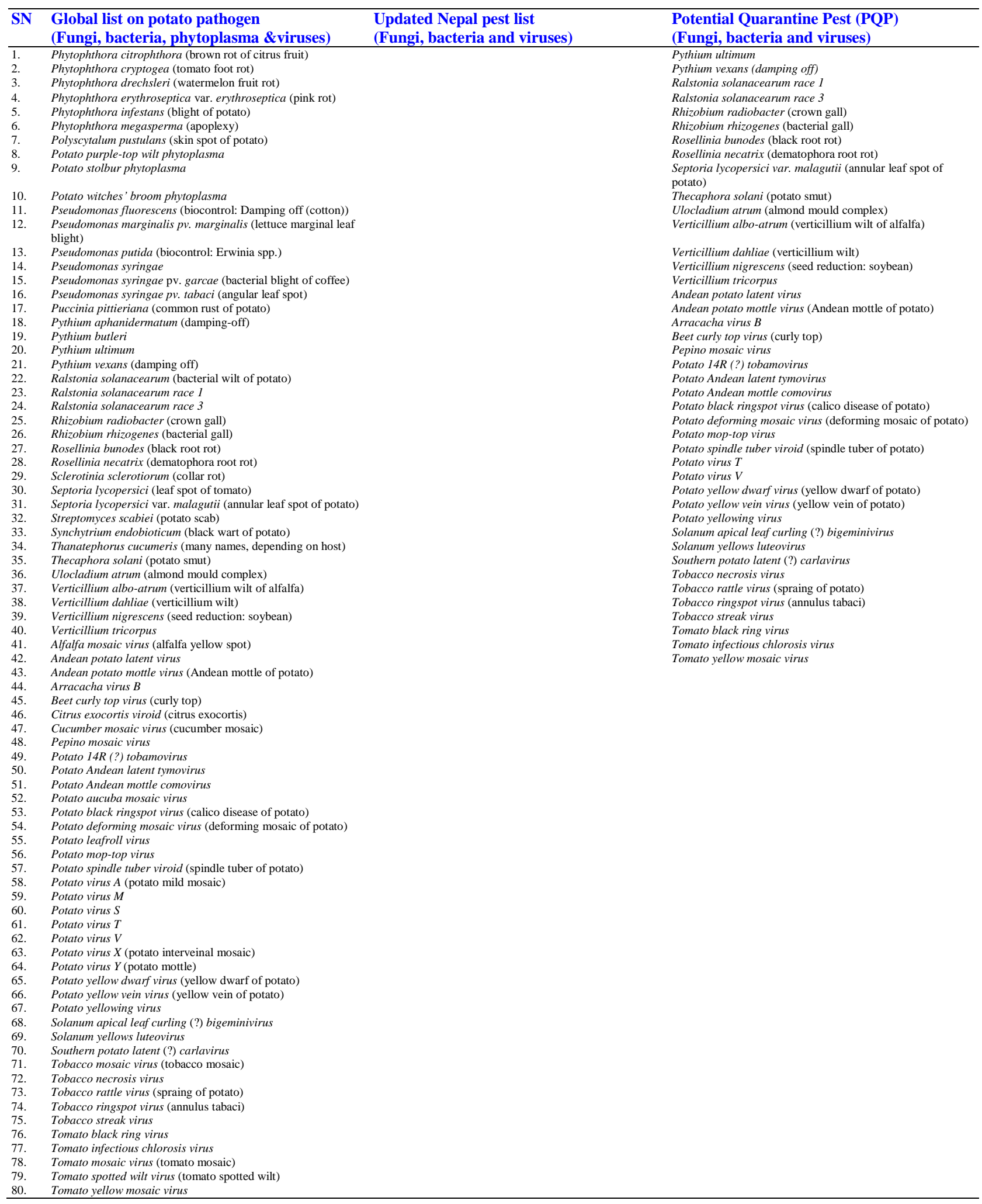




\section{RESULTS}

The data showed that all together 92 pathogens (fungi, bacteria and phytoplasma, viruses and viroids) were recorded as potential quarantine pests (PQP) for pest risk analysis (PRA). Out of 92 pathogens, fungi were 52, bacteria and phytoplasma were 13 and virus and viroids were 27 (Table 1). Detail information was not available for 15 pathogens. Among 15 pathogens fungi was 9, phytoplasma 1 and virus and viroids were 5. Out of 92 PQP for pest risk assessment, 34 were at high, 30 were of medium and 13 were of lower risk. Fifteen pests had no information. Risk management revealed that thirty-five pathogens (pests) were in high category, 30 pathogens (pests) in medium, 12 pathogens (pests) in low category (Table 2). Fifteen had no risk information. Out of 92 pests, sixty-one pathogen (pest) required additional declaration, 16 do not required additional declaration while fifteen had no information (Table 2).

\section{DISCUSSION}

The common pathogen of potato reported in Nepal are late blight (Phytophthora infestans), bacterial wilt (Pseudomonas solanacearum), black scurf (Rhizoctonia solani) and tuber warts (Synchytrium endobioticum). In the last 30 years some recommendations made by Nepal Agricultural Research Council (NARC 1995, 1997) such as crop rotation, use of clean planting materials could control or minimize the bacterial wilt incidences.

Virus is the main reason for the decline in potato crop production. When the seed lot is planted for many years, the yield potential of the variety reduced due to viral diseases. Depending on the type of virus, the yield might drops below $80 \%$. Degradation studies in the eastern terai showed that the virus infection decreases the tuber yield by $3.6 \%$ for every $10 \%$ increase in the infection (Wells et al 1996, Khairgauli 2054).

Mechanically transmitted virus such as potato virus $\mathrm{x}(\mathrm{PVX})$ is more common problem in the hills than in terai. Moreover, aphids spreading virus disease such as PVY is more common in plain areas. Other viruses such as PVM and PVS are found both in hills and plain areas (Shrestha 1997, Lama et al 1996, PRP 2003). The sanitary and phytosanitary (SPS) agreement requires that the disease database is must and the pest risk analysis (PRA) for the exchanged material is mandatory for international trade and commerce (FAO 2003).

But in Nepalese context information on various aspects such as entry, establishment, biology, epidemiology and economic impact is not sufficient for exchange of genetic materials. Crop Protection Compendium (CPC 2006) was used as a reference for information before.

Table 2. List of PQP and their categories, risk assessment, management and additional declaration

\begin{tabular}{|c|c|c|c|c|c|}
\hline $\mathbf{S N}$ & Potential Quarantine Pest & Category & $\begin{array}{l}\text { Risk } \\
\text { Assessment } \\
\text { Rating }\end{array}$ & $\begin{array}{l}\text { Risk } \\
\text { Management } \\
\text { Rating }\end{array}$ & $\begin{array}{l}\text { Additional } \\
\text { Declaration }\end{array}$ \\
\hline 1. & Aecidium cantense (Aecidium potato rust) & Fungi & medium & medium & Not required \\
\hline 2. & Alternaria radicina (black rot of carrots) & Fungi & medium & low & Not required \\
\hline 3 . & Arthrinium phaeospermum & Fungi & No information & No information & No information \\
\hline 4. & Belicobasidium purpureum Pat. & Fungi & No information & No information & No information \\
\hline 5. & Botryotinia fuckeliana (Botrytis rot) & Fungi & medium & medium & Not required \\
\hline 6. & Chalara elegans (black root rot) & Fungi & medium & medium & Not required \\
\hline 7. & Choanephora cucurbitarum (Choanephora blossom blight) & Fungi & low & medium & Required \\
\hline 8. & Cochliobolus australiensis & Fungi & medium & medium & Required \\
\hline 9. & Cochliobolus hawaiiensis (leaf spot: maize) & Fungi & medium & medium & Required \\
\hline 10. & Coleosporium tussilaginis f.sp. senecionis-sylvati & Fungi & No information & No information & No information \\
\hline 11. & Colletotrichum coccodes (anthracnose of tomato) & Fungi & No information & No information & No information \\
\hline 12. & Didymella bryoniae (gummy stem blight) & Fungi & medium & High & Required \\
\hline 13. & Didymella lycopersici (canker of tomato) & Fungi & low & medium & Not required \\
\hline 14. & Erysiphe orontii (powdery mildew) & Fungi & high & medium & Required \\
\hline 15. & Fusarium & Fungi & low & low & Not required \\
\hline 16. & Fusarium culmorum (culm rot: cereals) & Fungi & low & medium & Required \\
\hline 17. & Fusarium oxysporum f.sp. tuberosi & Fungi & medium & medium & Required \\
\hline 18. & Geotrichum candidum (sour rot: Citrus spp.) & Fungi & medium & medium & Required \\
\hline 19. & Gibberella avenacea (bud rot) & Fungi & medium & High & Required \\
\hline 20. & Gibberella baccata (biocontrol: velvetleaf) & Fungi & low & low & Not required \\
\hline 21. & Gibberella zeae (cobweb disease) & Fungi & low & low & Not required \\
\hline 22. & Helminthosporium solani (silver scurf) & Fungi & high & High & Required \\
\hline 23. & Hypocrea rufa (fruit rot: Citrus spp.) & Fungi & low & low & Not required \\
\hline 24. & Melanotus communis (wireworm, common) & Fungi & low & low & Not required \\
\hline 25. & Nectria haematococca (dry rot of potato) & Fungi & medium & medium & Not required \\
\hline 26. & Phacidiopycnis tuberivora (dryrot: potato) & Fungi & No information & No information & No information \\
\hline 27. & Phialophora parasitica (wilt: date palm) & Fungi & No information & No information & No information \\
\hline 28. & Phoma andigena (black blight of potatoes) & Fungi & medium & medium & required \\
\hline
\end{tabular}


Pest risk analysis in potato by BN Mahto

\begin{tabular}{|c|c|c|c|c|c|}
\hline $\mathbf{S N}$ & Potential Quarantine Pest & Category & $\begin{array}{l}\text { Risk } \\
\text { Assessment } \\
\text { Rating } \\
\end{array}$ & $\begin{array}{l}\text { Risk } \\
\text { Management } \\
\text { Rating } \\
\end{array}$ & $\begin{array}{l}\text { Additional } \\
\text { Declaration }\end{array}$ \\
\hline 1. & Phoma eupyrena & Fungi & medium & medium & Required \\
\hline 2. & Phoma exigua var. exigua (leaf blotch) & Fungi & low & low & Not required \\
\hline 3. & Phoma foveata (potato gangrene) & Fungi & medium & medium & Required \\
\hline 4. & Phytophthora citrophthora (brown rot of citrus fruit) & Fungi & low & low & Not required \\
\hline 5. & Phytophthora cryptogea (tomato foot rot) & Fungi & medium & low & Not required \\
\hline 6. & Phytophthora drechsleri (watermelon fruit rot) & Fungi & high & High & Required \\
\hline 7. & Phytophthora erythroseptica var. erythroseptica (pink rot) & Fungi & high & High & Required \\
\hline 8. & Phytophthora megasperma (apoplexy) & Fungi & high & High & Required \\
\hline 9. & Polyscytalum pustulans (skin spot of potato) & Fungi & high & High & Required \\
\hline 10. & Puccinia pittieriana (common rust of potato) & Fungi & high & High & Required \\
\hline 11. & Pythium butleri & Fungi & high & High & Required \\
\hline 12. & Pythium ultimum & Fungi & high & High & Required \\
\hline 13. & Pythium vexans (damping off) & Fungi & medium & medium & Required \\
\hline 14. & Rhizobium radiobacter (crown gall) & Fungi & high & High & Required \\
\hline 15. & Rhizobium rhizogenes (bacterial gall) & Fungi & high & High & Required \\
\hline 16. & Rosellinia bunodes (black root rot) & Fungi & No information & No information & No information \\
\hline 17. & Rosellinia necatrix (dematophora root rot) & Fungi & medium & medium & Not required \\
\hline 18. & Septoria lycopersici var. malagutii (annular leaf spot of potato) & Fungi & high & High & Required \\
\hline 19. & Thecaphora solani (potato smut) & Fungi & high & High & Required \\
\hline 20. & Ulocladium atrum (almond mould complex) & Fungi & medium & medium & Required \\
\hline 21. & Verticillium albo-atrum (verticillium wilt of alfalfa) & Fungi & high & High & Required \\
\hline 22. & Verticillium dahliae (verticillium wilt) & Fungi & high & High & Required \\
\hline 23. & Verticillium nigrescens (seed reduction: soybean) & Fungi & No information & No information & No information \\
\hline 24. & Verticillium tricorpus & Fungi & No information & No information & No information \\
\hline 25. & Bacillus polymyxa (bacterial fruit rot) & Bacteria & medium & medium & Required \\
\hline 26. & $\begin{array}{l}\text { Erwinia carotovora subsp. carotovora (bacterial root rot of sweet } \\
\text { potato) }\end{array}$ & Bacteria & high & High & Required \\
\hline 27. & Erwinia chrysanthemi & Bacteria & medium & medium & Required \\
\hline 28. & Erwinia chrysanthemi pv. chrysanthemi (bacterial maize stalk rot) & Bacteria & medium & medium & Required \\
\hline 29. & Pectobacterium chrysanthemi (slow wilt) & Bacteria & high & High & Required \\
\hline 30. & Pseudomonas fluorescens (biocontrol: Damping off (cotton)) & Bacteria & low & low & Not required \\
\hline 31. & $\begin{array}{l}\text { Pseudomonas marginalis pv. marginalis (lettuce marginal leaf } \\
\text { blight) }\end{array}$ & Bacteria & medium & medium & Required \\
\hline 32. & Pseudomonas putida (biocontrol: Erwinia spp.) & Bacteria & low & low & Not required \\
\hline 33. & Pseudomonas syringae pv. garcae (bacterial blight of coffee) & Bacteria & medium & medium & Required \\
\hline 34. & Pseudomonas syringae pv. tabaci (angular leaf spot) & Bacteria & medium & medium & Required \\
\hline 35. & Potato purple-top wilt phytoplasma & Phytoplasma & high & High & Required \\
\hline 36. & Potato stolbur phytoplasma & Phytoplasma & No information & No information & No information \\
\hline 37. & Potato witches' broom phytoplasma & Phytoplasma & high & High & Required \\
\hline 38. & Andean potato latent virus & Virus & high & high & required \\
\hline 39. & Andean potato mottle virus (Andean mottle of potato) & Virus & high & high & required \\
\hline 40. & Arracacha virus $B$ & Virus & medium & low & required \\
\hline 41. & Beet curly top virus (curly top) & Virus & medium & medium & required \\
\hline 42. & Pepino mosaic virus & Virus & medium & medium & required \\
\hline 43. & Potato $14 R(?)$ tobamovirus & Virus & no information & no information & no information \\
\hline 44. & Potato Andean latent tymovirus & Virus & high & high & required \\
\hline 45. & Potato Andean mottle comovirus & Virus & high & high & required \\
\hline 46. & Potato black ringspot virus (calico disease of potato) & Virus & high & high & required \\
\hline 47. & Potato deforming mosaic virus (deforming mosaic of potato) & Virus & high & high & required \\
\hline 48. & Potato mop-top virus & Virus & high & high & required \\
\hline 49. & Potato spindle tuber viroid (spindle tuber of potato) & Virus & high & high & required \\
\hline 50. & Potato virus $T$ & Virus & high & high & required \\
\hline 51. & Potato virus $V$ & Virus & no information & no information & no information \\
\hline 52. & Potato yellow dwarf virus (yellow dwarf of potato) & Virus & high & high & required \\
\hline 53. & Potato yellow vein virus (yellow vein of potato) & Virus & high & high & required \\
\hline 54. & Potato yellowing virus & Virus & high & high & required \\
\hline 55. & Solanum apical leaf curling (?) bigeminivirus & Virus & no information & no information & no information \\
\hline 56. & Solanum yellows luteovirus & Virus & no information & no information & no information \\
\hline 57. & Southern potato latent (?) carlavirus & Virus & no information & no information & no information \\
\hline 58. & Tobacco necrosis virus & Virus & medium & medium & required \\
\hline 59. & Tobacco rattle virus (spraing of potato) & Virus & high & high & required \\
\hline 60. & Tobacco ringspot virus (annulus tabaci) & Virus & medium & medium & required \\
\hline 61. & Tobacco streak virus & Virus & medium & medium & required \\
\hline 62. & Tomato black ring virus & Virus & medium & medium & required \\
\hline 63. & Tomato infectious chlorosis virus & Virus & high & high & required \\
\hline 64. & Tomato yellow mosaic virus & Virus & medium & medium & required \\
\hline
\end{tabular}

\section{CONCLUSION}

In generic PRA, a total of ninety-two potential quarantine pests (fungi, bacteria and phytoplasma, viruses and viroids) have been included. However, detail information on 15 pests is unavailable. A PRA should be sufficiently documented, so that when dispute arises, the PRA should clearly help to make decision on phytosanitary measures to be taken.

\section{REFERENCES}

CPC 2006. Crop Protection Compendium. CABI.

FAO. 2003. Database generation and pest risk analysis. Strengthening Plant Quarantine Services in Nepal TCP/NEP 2903. Working Paper, FAO, Kathmandu. 
Jha RK, SL Joshi, BN Mahato and DS Poudyal. 2006. Generic Pest Risks Analysis of Trade Priority Commodities: Capability and Constraints in Nepal. In: Proceedings of a National Workshop on Integrated Pest Management (IPM). Plant Protection Society Nepal, pp.236-252.

Khairgauli LP. 2054. Aalu bali. Aalu Post Project Sahyog, Aalu bali anusandhan karyakram, Swiss Sarkar Bikash Sahyog, Kathmandu (in Nepali language).

Lama TL GP Rai and BB Khatri. 1996. Potato program in Nepal: Collaboration with International Potato Centre (CIP). Paper presented in: Seminar on collaboration of International Potato Centre (CIP) in South West Asia Region, $25^{\text {th }}$ Anniversary celebration, 2-3 Dec 1996, Kathmandu, Nepal.

MoAD. 2013. National standards for phytosanitary measures, Ministry of Agriculture Development, Kathmandu, Nepal.

NARC. 1995. The Research Priorities Approaches and Support need for Agricultural Research. Paper presented in: Donors Consortium Meeting held on 25 April 1995.

NARC. 1997. 25 Years of Potato Research in Nepal (1972-1997). Silver Jubilee, NARC, Potato Research Programme, Nepal.

PRP. 2003. Annual Report. Potato Research Program, Lalitpur, Nepal.

Shrestha SK. 1997. Potato diseases in Nepal. Nep. J. Agric. 13/14:36.

Ullah Md A. 2016. Pest Risk Analysis (PRA) of Brinjal in Bangladesh. Strengthening Phytosanitary Capacity in Bangladesh Project Plant Quarantine Wing Department of Agricultural Extension Khamarbari, Farmgate, Dhaka-1205, Bangladesh.

Wells GJ, S Schulz and MRanjit. 1996. Final report of the national potato research and development programmes Phase IV, Swiss Agency for Development and Cooperation, Nepal. 